\title{
The spiral galaxy M 33 mapped in the FIR by ISOPHOT
}

\section{A spatially resolved study of the warm and cold dust ${ }^{\star}$}

\author{
H. Hippelein ${ }^{1}$, M. Haas ${ }^{1}$, R. J. Tuffs ${ }^{2}$, D. Lemke ${ }^{1}$, M. Stickel ${ }^{1}$, U. Klaas ${ }^{1}$, and H. J. Völk ${ }^{2}$ \\ 1 Max-Planck-Institut für Astronomie, Königstuhl 17, 69117 Heidelberg, Germany \\ 2 Max-Planck-Institut für Kernphysik, Saupfercheckweg, 69117 Heidelberg, Germany
}

Received 28 March 2003 / Accepted 20 May 2003

\begin{abstract}
The Sc galaxy M 33 has been mapped with ISOPHOT in the far-infrared, at 60, 100, and $170 \mu \mathrm{m}$. The spatial resolution of these FIR maps allows the separation of spiral arms and interarm regions and the isolation of a large number of star-forming regions. The spectral energy distribution in the FIR indicates a superposition of two components, a warm one originating from dust at $\sim 45 \mathrm{~K}$, and a cold one, at $\sim 16 \mathrm{~K}$. The warm component is concentrated towards the spiral arms and the star-forming regions, and is likely heated by the UV radiation from OB stars. The cold component is more smoothly distributed over the disk, and heated by the diffuse interstellar radiation. For the about 60 star-forming regions detected the $\mathrm{H} \alpha /$ FIR flux ratio increases significantly with the distance from the galaxy center, probably due to decreasing extinction. An anti-correlation of $F_{\mathrm{H} a} / F_{60}$ with $F_{170}$ suggests the intrinsic extinction to be related to the cold dust surface brightness according to $A_{V} / S_{170} \sim 0.03 \mathrm{mag} \mathrm{MJy}^{-1}$ sr. For the total galaxy the star formation rate (SFR) derived from the FIR is in agreement with that derived from the de-extincted $\mathrm{H} \alpha$ emission. For individual star-forming regions, a consistency between SFRs derived from the optical and from the FIR requires only a fraction of the UV radiation to be absorbed locally. The individual star-forming regions also show a local radio-FIR correlation. This local correlation is, however, due to quite different components than to those that lead to the well-known global radio-FIR correlation for entire galaxies.
\end{abstract}

Key words. galaxies: spiral - galaxies: ISM

\section{Introduction}

The sensitivity of ISO (Kessler et al. 1996) and its spectral coverage extending to $200 \mu \mathrm{m}$ made it the first observatory capable of measuring the bulk of starlight absorbed by dust in galaxies. Recent FIR studies of normal galaxies (e.g. Haas et al. 1998 for M 31; see also Tuffs \& Popescu 2003 for a review), based on data taken with the ISOPHOT instrument (Lemke et al. 1996) onboard ISO, have proven the existence of a cold dust component with a temperature below $20 \mathrm{~K}$, in addition to the warm component near $T \sim 50 \mathrm{~K}$. These components were originally proposed by Chini et al. (1986), based on sub$\mathrm{mm}$ observations. Statistically significant evidence for the existence of a cold dust component was also inferred from the ISOPHOT Serendipity Survey (Stickel et al. 2000), and from the analysis of a complete volume- and luminosity limited sample of late-type Virgo Cluster galaxies - the ISOPHOT Virgo Cluster Deep Sample (Tuffs et al. 2002; Popescu et al. 2002). Even ultra-luminous IR galaxies show evidence for a cold dust

Send offprint requests to: $\mathrm{H}$. Hippelein,

e-mail: hippelei@mpia-hd.mpg.de

* Based on observations with the Infrared Space Observatory ISO. ISO is an ESA project with instruments funded by ESA Member States (especially the PI countries France, Germany, The Netherlands and the UK) and with the participation of ISAS and NASA. component (Klaas et al. 2001), though this does not constitute the bulk of the FIR emission, as in the case of normal galaxies. This cold component arises from grains which are in weaker radiation fields, either in the outer optically thin regions of the galaxy disks or because they are shielded from radiation by optical depth effects (Tuffs \& Popescu 2003).

Though the existence of the warm and cold components were unambiguously inferred from the statistical studies mentioned above, the spatial distributions of these components can only be studied by the analysis of resolved nearby galaxies. Such an analysis was done for the ISOPHOT map of M 31 by Haas et al. (1998) and Schmidtobreick et al. (2000), who resolved several dozens of bright knots and separated their SEDs in cold and warm dust components. While the FIR emission in M31, a Sab galaxy seen at high inclination, originates mainly from a ring-like structure rather than from spiral arms, M 33, a Sc galaxy with well distinct spiral arms, and seen almost face on, seems to be an ideal target to study the spatial distribution of dust and its heating sources in spiral galaxies. Due to its distance of only $830 \mathrm{kpc}$, its scale of $0.24 \mathrm{kpc} \mathrm{arcmin}^{-1}$ is sufficient to allow a separation of its constituents (nucleus, H II regions, spiral arms, and interarm regions) with ISOPHOT even at $170 \mu \mathrm{m}$.

We will make use of the higher spatial resolution and the extended spectral coverage (to longer FIR wavelengths) of 
ISOPHOT as compared with IRAS to disentangle the warm and cold dust components in M33. The spatial distributions of these dust components will be compared with the distribution of the optical emission. The FIR emission from individual HII regions will be correlated with the corresponding radio emission. In this paper we will also study the relation between FIR emission and star formation rate, as well as the heating of the two dust emission components and their effects on extinction.

\section{Observations and data reduction}

\subsection{FIR data}

M33 was mapped with ISOPHOT (Lemke et al. 1996) in the raster scan mode, AOT-P32, at three wavelengths, 60, 100, and $170 \mu \mathrm{m}$, using the $\mathrm{C} 100$ and $\mathrm{C} 200$ detector arrays. The raster step sizes in spacecraft coordinates were $69^{\prime \prime} \times 92^{\prime \prime}$ for 60 and $100 \mu \mathrm{m}$ and $92^{\prime \prime} \times 92^{\prime \prime}$ at $170 \mu \mathrm{m}$. Combined with the detector geometry and the motion of the focal plane chopper, this yielded a sky sampling of $15^{\prime \prime} \times 23^{\prime \prime}$ and $31^{\prime \prime} \times 92^{\prime \prime}$ respectively. Since the linear extension of M 33 is too large to be coverable by one raster map with the desired resolution, two partial maps had to be done in each filter (North and South) and concatenated. With the partial Northern and Southern P32 maps mosaiced together according to their center positions and satellite roll angles, a total area of $48^{\prime} \times 32^{\prime}$ was covered, including the entire disk of M 33, and a surrounding background area to allow for proper sky subtraction. The orientation of this map is roughly along the major axis of the galaxy.

At $170 \mu \mathrm{m}$ the central area of $29^{\prime} \times 26^{\prime}$ size was also mapped in the AOT-P22 raster staring mode, which almost is free from detector transient effects due to the relatively long integrating time of 10 seconds per raster position. The P32 and P22 maps were merged to a common one by using equal weights for both maps in the bright and medium bright areas and zero-weight for the P22 map for areas where the surface brightness falls below $13 \mathrm{MJy} / \mathrm{sr}$.

The data reduction was done using the ISOPHOT Interactive Analysis software package PIA V7 (Gabriel et al. 1998), together with the calibration data set V4.0 (Laureijs et al. 2000). A special data handling was required due to signal transient effects of the infrared detectors, which is described in Sect. 2.2. The absolute flux calibration was performed with measurements of the internal thermal fine calibration source on board, before and after each map. We estimate the error of this flux calibration to be about $\pm 20 \%$. An additional uncertainty is introduced by the detector transient effects in the P32 observing mode, which generally leads to too low signals for the FIR knots, and which we have only corrected to a certain degree (see below). At 60 and $100 \mu \mathrm{m}$, where this effect is most critical, we estimate the total error to be $\pm 30 \%$. At $170 \mu \mathrm{m}$ the transient effect is less severe, and the total error is of the order of $\pm 20 \%$.

\subsection{Handling of transient detector effects}

As mentioned above, a difficulty in the reduction of P32 data are signal transient effects of the infrared detectors, leading to small lags of the maxima positions for bright point-like sources along the scan direction, and to a lowering of the maxima and filling up of the minima. It also led to ghost images of bright sources at distances of the chopper throw in the scan direction. Based on the principles described by Acosta-Pulido et al. (2000) we adopted a correction routine to reduce the transient effects:

First, separate maps were constructed for each detector pixel, nine for the 60 and $100 \mu \mathrm{m}$ maps, which were observed with the $\mathrm{C} 100$ detector array. and four in the case of the $170 \mu \mathrm{m}$ map, which was observed with the C200 array. Transient effect were reduced by adding to the signal in each pixel $30 \%$ of the difference to the signal of the preceeding pixel during the scan. This procedure led to a steepening of the edges of bright sources and improved the coincidence in the forth and back scans. It also led to a significant reduction of the ghosts from bright point like sources at the map pixels located in chopper throw separation (here, however, a factor of only $10 \%$ was applied to the difference signal).

Second, the separate pixel maps were scaled to a common level and superimposed. Due to a displacement of the raster legs by half of the array size and a chopper step of $15^{\prime \prime}$ the pixel size of the resulting image was $15^{\prime \prime} \times 23^{\prime \prime}$ (in the cases of 60 and $100 \mu \mathrm{m})$. Each of these pixels was seen by 3 detector pixels in the one raster leg in forth direction and in the adjacent raster leg in opposite direction by 3 other detector pixels. From the 6 detector signals in each pixel the median signal was derived, and an iterative $\kappa-\sigma$ clipping was applied to the separate pixel maps and to correct them. This also leads to a spatial resolution of $60^{\prime \prime}$ at $60 \mu \mathrm{m}$, of $80^{\prime \prime}$ at $100 \mu \mathrm{m}$, and of $120^{\prime \prime}$ at $170 \mu \mathrm{m}$. It was found that each scan had a slight positive slope, recognizable by small offsets at the edges of the forth and back scans. This was corrected by a fine adjustment of the scan slopes until a smooth background was achieved. The background level (from foreground cirrus and zodiacal light) was then determined from the outermost map areas and subtracted.

\subsection{Optical observations}

For studying the correlation of optical emission with the detailed ISOPHOT maps, M 33 was observed in $\mathrm{H} \alpha$ with the focal reducer at the $2.2 \mathrm{~m}$ telescope on Calar Alto, Spain. The detector was a $2 \mathrm{k} \times 2 \mathrm{k}$ SITe chip used in a rebinned mode, to provide a scale of $1^{\prime \prime} 06$ pixel $^{-1}$, thus covering a field of $15^{\prime}$ diameter. The photometric conditions were good. The seeing of 2 '. 5 was adequate for this study. For isolating the $\mathrm{H} \alpha$ line emission a $5.2 \mathrm{~nm}$ wide filter, centered at $657.0 \mathrm{~nm}$ was used. For continuum subtraction we used a broad band red filter, centered at $650 \mathrm{~nm}$. To cover the whole galaxy, a mosaic of 13 positions was obtained. The single maps were corrected for the image distortion of the focal reducer (with flux conservation) and then aligned using stellar DSS positions and merged to an $\mathrm{H} \alpha$ map of $55^{\prime} \times 35^{\prime}$ size. The flux calibration for the $\mathrm{H} \alpha$ map was done using the total line flux value given by Devereux et al. (1997) and using the $\mathrm{H} \alpha$ line fluxes of the prominent $\mathrm{H}$ II regions tabulated in Hippelein (1986), assuming a mean relative intensity of the [NII] lines of 20\% (McCall et al. 1985). 

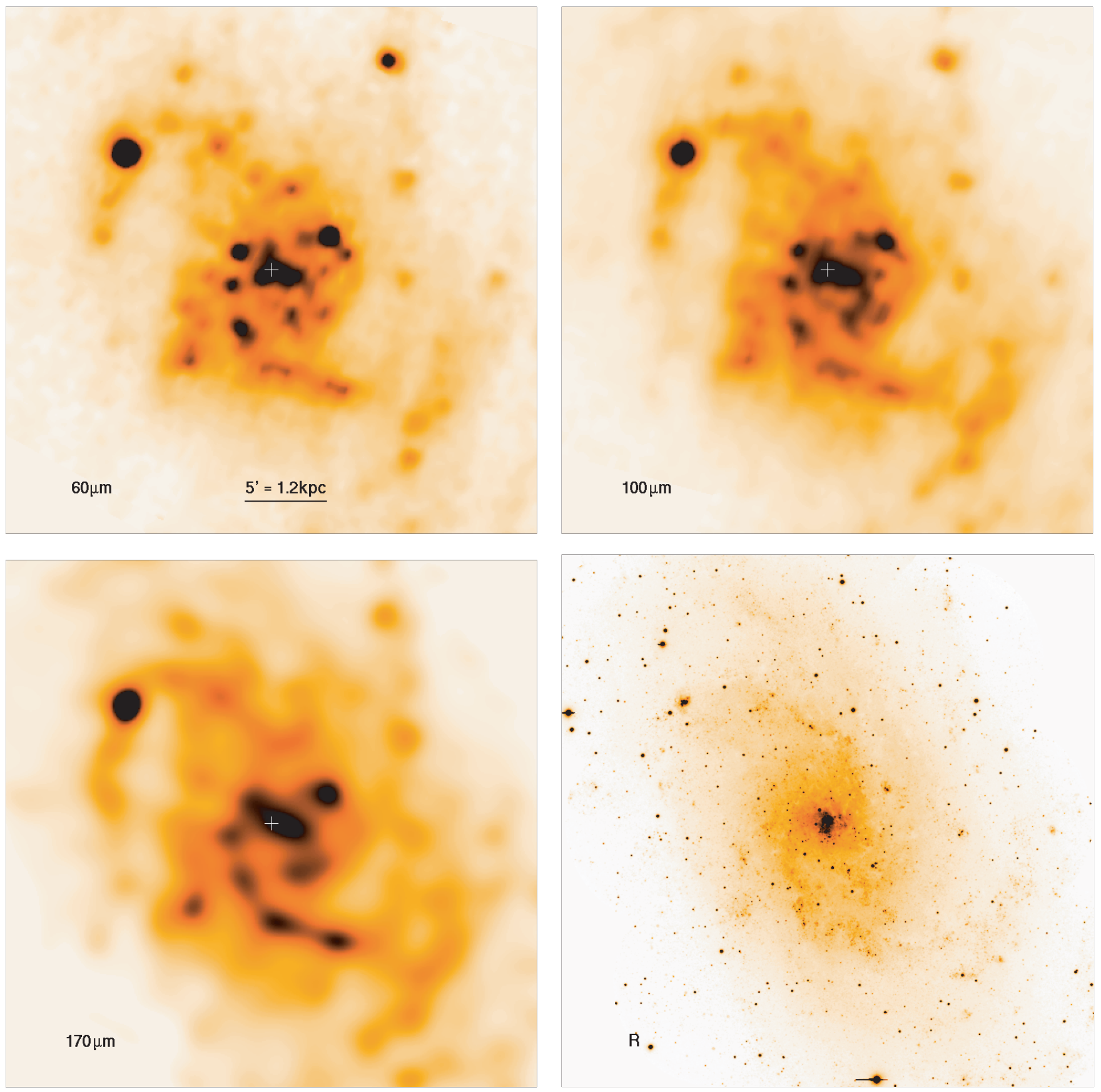

Fig. 1. FIR maps of M 33 (area $33^{\prime} \times 33^{\prime}$, North is up, East is left) at 60 (left top), 100 (right top), and $170 \mu \mathrm{m}$ (left bottom). The $170 \mu \mathrm{m}$ map is restored to a spatial resolution of $100^{\prime \prime}$, while the resolutions at 60 and $100 \mu \mathrm{m}$ are $60^{\prime \prime}$ and $80^{\prime \prime}$ respectively. For comparison, an $R$ image of the galaxy is also shown.

\section{Results}

In this section we will first present the general morphology of M 33, then we present the FIR photometry of discrete sources and their FIR spectral properties.

\subsection{Morphology}

Figure 1 presents the maps obtained at the three FIR wavelengths, rebinned to a pixel size of $5^{\prime \prime}$. The spatial resolutions are $60^{\prime \prime}$ at $60 \mu \mathrm{m}$ and $80^{\prime \prime}$ at $100 \mu \mathrm{m}$, as derived from the profile of IC 133, the bright region in the NW edge. In the case of $170 \mu \mathrm{m}$ the spatial resolution of $\approx 120^{\prime \prime}$, as measured from the profile of NGC 604 (IC 133 is too faint at $170 \mu \mathrm{m}$ ), was improved to $100^{\prime \prime}$ by deconvolving the original map with a PSF of $120^{\prime \prime}$.

All three FIR maps clearly show the spiral arm structure of the galaxy and a large number of distinct sources. In addition to the spiral structure, an extended underlying component can be recognized, which becomes more significant with increasing wavelength. This is illustrated by the intensity profiles in Fig. 2, showing cuts through the three FIR maps at a position 


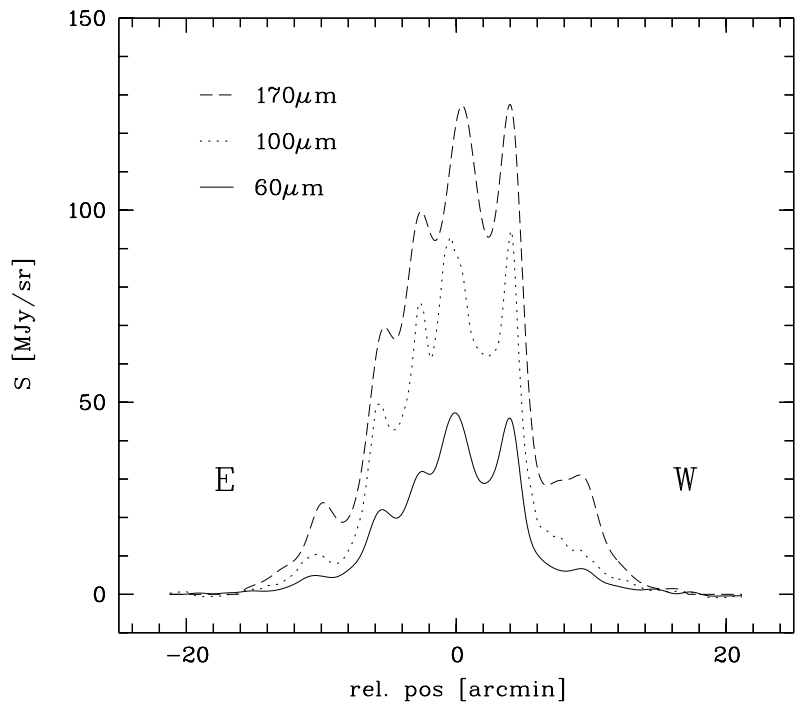

Fig. 2. Profiles through the center of M 33 along position angle $115^{\circ}$ for the three FIR wavelengths. Here, the $60 \mu \mathrm{m}$ map is convolved with a Gaussian of $60^{\prime \prime} F W H M$ to achieve a similar spatial resolution as the $100 \mu \mathrm{m}\left(80^{\prime \prime} F W H M\right)$, and the $170 \mu \mathrm{m}$ profile is taken from the Lucy-Richardson restored map.

angle of $115^{\circ}$, along the minor axis. The structure of the profiles is identical for the three filters, except that at the edge of the galaxy the $170 \mu \mathrm{m}$ profile seems to decay slower than the others.

The total flux density of M 33 is $560 \mathrm{Jy}, 1250 \mathrm{Jy}$, and $2200 \mathrm{Jy}$ at 60,100 , and $170 \mu \mathrm{m}$, respectively.

\subsection{FIR photometry of discrete sources}

About sixty sources were isolated from the FIR maps shown in Fig. 1. This number is large enough to allow statistical studies for the FIR properties of extragalactic star-forming regions. Aperture photometry was done for these sources, with aperture diameters between $100^{\prime \prime}$ for small regions, up to $260^{\prime \prime}$ for the giant star-forming region NGC 604. Since in the crowded areas the diffuse emission from M33 cannot be unambigously determined, and since the introduction of a model for this component would be rather subjective and lead to arbitrary results, the photometry is performed without a subtraction of the diffuse emission.

From IRAS maps of M33, Rice et al. (1990) localized a number of $\mathrm{H}$ II complexes and derived flux densities at 60 and $100 \mu \mathrm{m}$. Their $60 \mu \mathrm{m}$ flux densities (Table 1) are in reasonable agreement with ours, while their $100 \mu \mathrm{m}$ data are generally higher, probably due to the inferior spatial resolution of IRAS.

\subsection{Spectral energy distribution}

Figure 3 depicts the SEDs as derived from the photometry for a number of selected areas. In the cases of the total galaxy and of NGC 604 and NGC 595 the IRAS (Rice et al. 1990) points are overplotted. The COBE/DIRBE (Odenwald et al. 1998) data overplotted in the SED for the total galaxy are somewhat lower. The explanation for this difference is that M 33 is situated in a
Table 1. Comparison with IRAS results of Rice et al. (1990). $\Phi$ are the aperture diameters used for the ISO photometry, $F_{60}$ stands for flux density $F_{v}$ at $60 \mu \mathrm{m}$ in Jy, etc.

\begin{tabular}{|c|c|c|c|c|c|}
\hline Name & \multicolumn{3}{|c|}{ ISOPHOT } & \multicolumn{2}{|c|}{ IRAS } \\
\hline total & & 560 & 1250 & 420 & 1260 \\
\hline В 651 & $100^{\prime \prime}$ & 1.0 & 1.9 & 1.7 & 4.5 \\
\hline IC 133 & $140^{\prime \prime}$ & 6.3 & 7.3 & 11.8 & 10.7 \\
\hline IK 60 & $120^{\prime \prime}$ & 2.4 & 4.6 & 2.4 & $\ldots$ \\
\hline NGC 604 & $260^{\prime \prime}$ & 29.5 & 46.4 & 33.6 & 54.5 \\
\hline IC1 31 & $140^{\prime \prime}$ & 3.2 & 6.4 & 2.7 & 3.2: \\
\hline NGC 595 & $140^{\prime \prime}$ & 13.0 & 22.9 & 12.8 &... \\
\hline B $220 / 1$ & $100^{\prime \prime}$ & 2.4 & 5.4 & 1.9 & ... \\
\hline В $255 / 7$ & $100^{\prime \prime}$ & 1.7 & 4.1 & 1.6 & $\ldots$ \\
\hline В 248-51 & $120^{\prime \prime}$ & 2.6 & 5.5 & 2.7 & 8.3 \\
\hline
\end{tabular}

small hole in the cirrus foreground, and the off-galaxy background measured by COBE/DIRBE with its large beam in the neighbourhood is probably too high, leading to lower flux densities for the galaxy.

In all cases (Fig. 3) it is obvious from the distribution of the three FIR data points that the SEDs cannot be represented by a single blackbody, but need a combination of two blackbody emission components. We associate these two emissions with the two main morphological components measured directly in this paper, namely the localised (mainly warm) and the diffuse (mainly cold) component. Two blackbodies have four degrees of freedom and as there are only three data points, no unique combination can be derived. In the cases of M33(total) and NGC 604 however, the IRAS data point at $25 \mu \mathrm{m}$ puts an upper limit to the temperature of the warm blackbody component.

At the long wavelength end Chini et al. (1986) found for $\mathrm{Sb}$ and $\mathrm{Sc}$ type galaxies observed by IRAS an average ratio $S_{1200 \mu \mathrm{m}} / S_{60 \mu \mathrm{m}} \sim 0.034$, with a scatter of a factor 2.5 . Adopting the same ratio for M 33 we insert a $1200 \mu \mathrm{m}$ data point into the M 33 SED.

Attributing $30 \%$ of the signal at $25 \mu \mathrm{m}$ to hot dust, the fit with two modified blackbody functions (assuming an emissivity of $\beta \propto \lambda^{-2}$ ) to the four data points between 25 and $170 \mu \mathrm{m}$ yields $T_{\text {warm }}=46.0 \pm 0.8 \mathrm{~K}$, and $T_{\text {cold }}=16.7 \pm 0.5 \mathrm{~K}$. As seen in the plot the cold blackbody curve comes very close to the $1200 \mu \mathrm{m}$ point estimated above. Adopting the temperature of the warm component to be rather constant over the galaxy, we keep $T_{\text {warm }}=46 \mathrm{~K}$ fixed for fitting the cold component in the other areas. The resulting $T_{\text {cold }}$ varies only slightly, taking values between 15.3 and $17.1 \mathrm{~K}$, as demonstrated in the figure, with error bars of typically $\pm 0.5 \mathrm{~K}$. This indicates that (coincidentially) the temperature of the diffuse cold dust component is rather close to the temperature of the localised cold dust component. If one does the same for $\beta \propto \lambda^{-1}$, one would get $T_{\text {warm }}=52.5 \pm 1.0 \mathrm{~K}$, and $T_{\text {cold }}=19.4 \pm 0.7 \mathrm{~K}$ for $\mathrm{M} 33_{\text {total }}$, with a submm value a factor of 3 above the adopted one (dotted curve), while the variation of $T_{\text {cold }}$ was again rather small over the field.

Therefore, we interpret the spatial variation of the flux density ratio $F_{\text {warm }} / F_{\text {cold }}$ as being due to a variation of their 

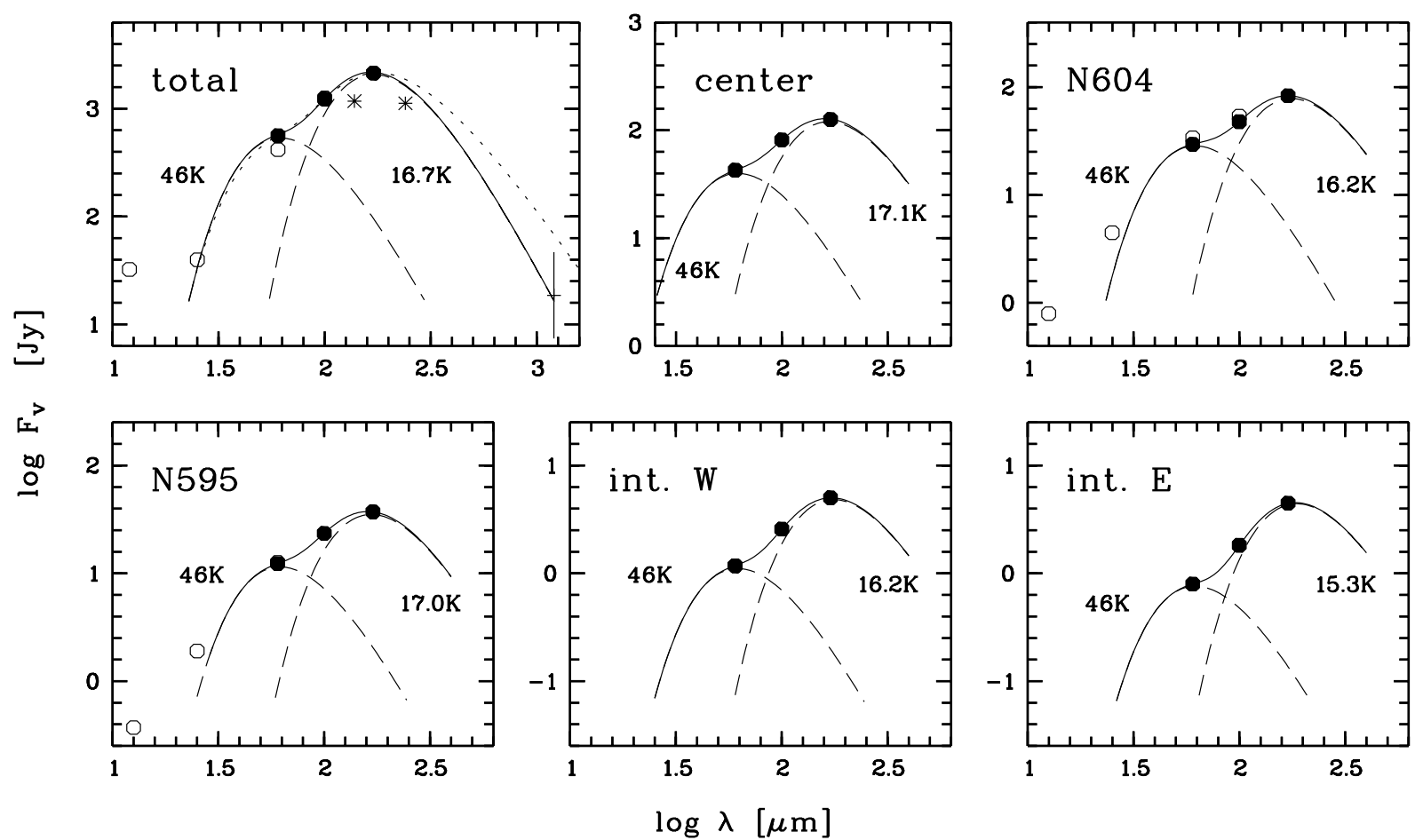

Fig. 3. Spectral energy distribution for total M 33 (top left), for the central area (top center), for two prominent H II regions (NGC 604, NGC 595), and for two areas in the interarm regime, W int. (offset $6^{\prime} \mathrm{W}, 5^{\prime} \mathrm{S}$ from the nucleus) and int. E ( $8^{\prime} \mathrm{E}$ from the nucleus). Dots are ISOPHOT data, circles stand for IRAS, asterisks for COBE/DIRBE data, and the plus with the large error bar at $1200 \mu \mathrm{m}$ is derived from flux density ratios as estimated by Chini et al. 1986). Two-component blackbody fits for temperatures of $T_{\text {warm }}=46.0 \mathrm{~K}, T_{\text {cold }} \sim 16.5 \mathrm{~K}$, respectively, and with emissivity $\beta \propto \lambda^{-2}$ are overlaid as dashed and solid lines. For comparison, the dotted line shows a fit for $\beta \propto \lambda^{-1}$ with $T_{\text {warm }}=52.0 \mathrm{~K}, T_{\text {cold }}=19 \mathrm{~K}$.

amplitudes, rather than being due to a change of their temperatures, which seem to remain almost constant over the galaxy. Since the warm and the cold component peak very close to 60 and $170 \mu \mathrm{m}$, respectively, the ISOPHOT 60 and $170 \mu \mathrm{m}$ flux densities can be used as tracers of the warm and the cold dust.

\section{Discussion}

\subsection{Spatial distribution of the warm dust}

In Fig. 4 , the ratios $F_{60} / F_{170}$ for all FIR knots are plotted versus the deprojected radial distance from the center of the galaxy. For the deprojection an inclination of $56^{\circ}$ and a position angle of PA $=23^{\circ}$ was assumed for the galaxy (Tully 1988). In addition a number of positions in the interarm regions (open circles) were selected. Firstly, it is obvious that the flux density ratio for the interarm areas is lower than that of the bright regions at the same radius. Secondly, both the ratios in the interarm and bright regions decrease with increasing radial distance, except for the bright H II regions NGC604 and IC 133. In the case of the interarm regions, this decrease can be explained by a decrease in the 60/170 micron colour temperature of the diffuse emission of M 33. In the case of the bright star-forming regions, the decrease in the $F_{60} / F_{170}$ ratio is attributed to an increasing relative contribution of the diffuse $170 \mu \mathrm{m}$ emission from the disk in the fainter, outer parts. Very bright regions, such as NGC604, but also those in the central area are almost unaffected by this diffuse disk component. A special case is
IC 133 due to its extraordinarily high $F_{60}$ value. Schulman \& Bregman (1995) found it associated with a bright X-ray source located in a hole in the HI layer of the galaxy, indicating energetic stellar winds and supernovae from massive stars. The average $F_{60} / F_{170}$ ratio for the 20 bright star-forming regions is 0.32 , with a dispersion of 0.05 , and 0.165 for the interarm regions with a scatter of 0.03 .

The observed FIR brightness distribution is a superposition of localized emission from star-forming regions and emission from a diffuse component. The localized FIR emission is mainly powered by UV photons, whereas the diffuse FIR emission is powered by both UV and optical photons. Models of the UV-optical/FIR-submm SEDs which self consistently calculate a continuous distribution of dust temperatures based on radiative-transfer calculations (see Popescu \& Tuffs 2002a) predict that, for $\lambda<100 \mu \mathrm{m}$, the FIR emission is dominated by the localized component. These models also predict that at longer wavelengths most emission originates from the diffuse disk component, and that both the localized and the diffuse dust emissions are predominantly powered by the UV light.

We will try to separate the localized FIR emission (from star-forming regions) from the diffuse FIR emission component. While for central distances larger than $r=8^{\prime}$ the interarm areas are wide enough to allow an estimate of the diffuse radiation from the disk, this is not possible in the inner area of the galaxy.

Two simple approaches are performed. Firstly, assuming an exponential disk with a central flux density of $25 \mathrm{MJy} / \mathrm{sr}$ at 


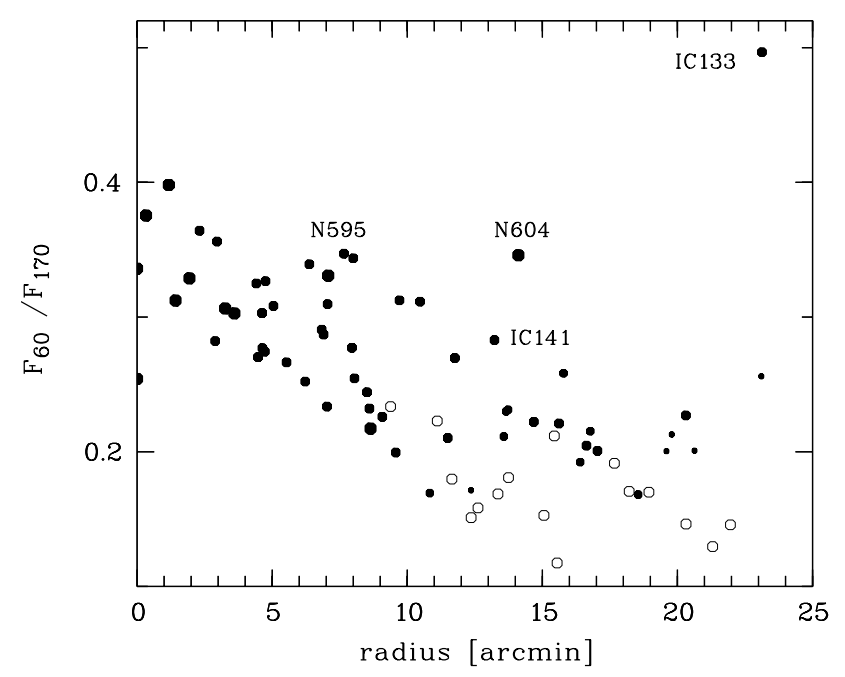

Fig. 4. The $F_{60} / F_{170}$ ratio versus deprojected distance from the galaxy center. Large dots are for star-forming regions with high $100 \mu \mathrm{m}$ flux density, small dots for faint ones. Some prominent regions are labeled by their names. Empty circles stand for inter-arm regions.

$60 \mu \mathrm{m}$ and a scale length of $6^{\prime}(1.44 \mathrm{kpc})$ for the diffuse emission component would yield a residual map for the localized emission with a level in the inter-arm regions close to zero. The contribution of the diffuse disk to the total $60 \mu \mathrm{m}$ radiation would be $42 \%$. This number agrees with the model for the $\mathrm{Sb}$ galaxy NGC 891 by Popescu et al. (2000) for which the contribution of the diffuse emission to the total is about $40 \%$ at $60 \mu \mathrm{m}$.

A second approach to obtain the contribution of the starforming regions at 60 microns is to make a linear combination of the 60 and $170 \mu \mathrm{m}$ maps. If we denote the diffuse emission component at 60 and 170 micron by $F_{60}^{\mathrm{d}}$ and $F_{170}^{\mathrm{d}}$, respectively, and correspondingly the localized emission components by $F_{60}^{1}$ and $F_{170}^{1}$, then the arm and interarm colour ratios obtained previously can be written as $f^{1}=F_{60}^{1} / F_{170}^{1}$ and $f^{\mathrm{d}}=F_{60}^{\mathrm{d}} / F_{170}^{\mathrm{d}}$, respectively. In this case the localized emission component at 60 micron is given by the following equation, with $F_{60}$ and $F_{170}$ in Jy:

$F_{60}^{1}=\frac{F_{60}-f^{\mathrm{d}} \times F_{170}}{1-f^{\mathrm{d}} / f^{\mathrm{l}}}$.

For $f^{\mathrm{d}}=0.165$ and $f^{1}=0.32$, we find that $80 \%$ of the emission at 60 micron comes from the localized component. So only $20 \%$ of the emission is diffuse at this wavelength. The $20 \%$ value is lower than that obtained with the first method. However this is to be expected, since here we neglected the radial dependence of the $f^{\mathrm{d}}$ and $f^{\mathrm{l}}$ factors. The result in Fig. 5 shows the star-forming regions well isolated against a zero-level background.

\subsection{Correlation of localized $60 \mu \mathrm{m}$ emission with $\mathrm{H} \alpha$ emission}

The location of the FIR knots is also traced in the distribution of H II regions, best seen in the optical, in the light of the $\mathrm{H} \alpha$ line. In order to correlate the dust emission heated by the UV

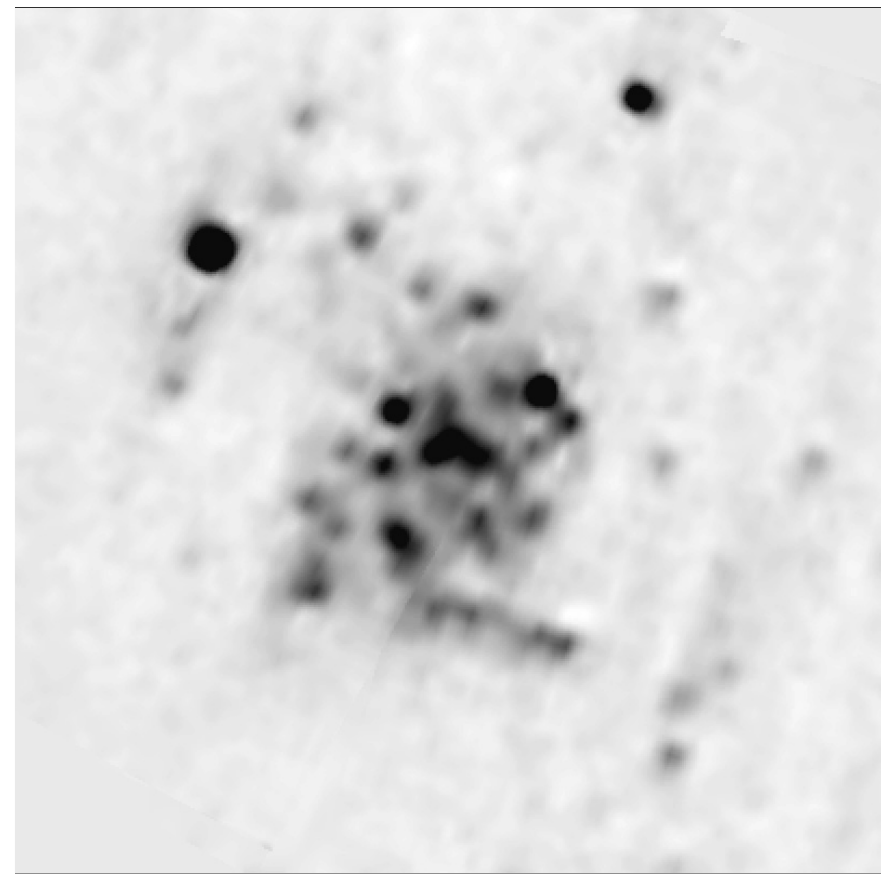

Fig. 5. Distribution of the localised warm dust component at $60 \mu \mathrm{m}$, $F_{60}^{\mathrm{l}}$, visualised by the scaled difference map $2\left(F_{60}-0.165 \times F_{170}\right)$, with the factor 0.165 given by the average flux density ratio $F_{60} / F_{170}$ in the inter-arm regions and the factor 2 given by Eq. (1).

radiation from star-forming regions with the $\mathrm{H} \alpha$ emission from the associated H II regions, we make use of the map in Fig. 6. Before, the $\mathrm{H} \alpha$ map needs to be convolved with a Gaussian profile of $60^{\prime \prime} F W H M$ to achieve a similar spatial resolution. Figure 6 shows the central $33^{\prime} \times 33^{\prime}$ area of this map. Its morphology is almost indistinguishable from the map of localised warm dust emission at $60 \mu \mathrm{m}, F_{60}^{1}$ (Fig. 5), except for the fact that the ratio of FIR to $\mathrm{H}_{\alpha}$ emission appears to be higher in the central area than in the outskirts.

The $F_{\mathrm{H} \alpha} / F_{60}^{1}$ ratio (Fig. 7 , where $F_{\mathrm{H} \alpha}$ is the flux in the line) for the FIR knots shows a clear systematic increase with increasing radial distance from the center (allowing for the [NII] line contribution decreasing with distance, the slope would be even steeper). The low data point at $r=$ 15 arcmin in Fig. 7 originates again from the star-forming region IC 133 with its unusually high $60 \mu \mathrm{m}$ flux density value. The least squares fit to the data points provides a slope of $\mathrm{d}\left(\log \left(F_{\mathrm{H} \alpha} / F_{60}^{1}\right)\right) / \mathrm{d}(r)=0.038 /$ arcmin.

A significant increase of the $F_{\mathrm{H} \alpha} / F_{\mathrm{FIR}}$ ratio towards the outer parts of the galaxy was also reported by Devereux et al. (1997). Their slope is however less steep, since it includes not only the star-forming regions but also locations inbetween the spiral arms, where the ratio is systematically lower.

\subsection{Extinction}

While the foreground extinction towards M33 is negligible $\left(A_{V}=0.09 \mathrm{mag}\right.$, de Vaucouleurs et al. 1991, or $A_{V}=$ 0.18 mag, Burstein \& Heiles 1982; Schlegel et al. 1998), Israel \& Kennicutt (1980) and Berkhuijsen (1983) derived from a comparison of $\mathrm{H} \alpha$ data with radio flux densities at 21 


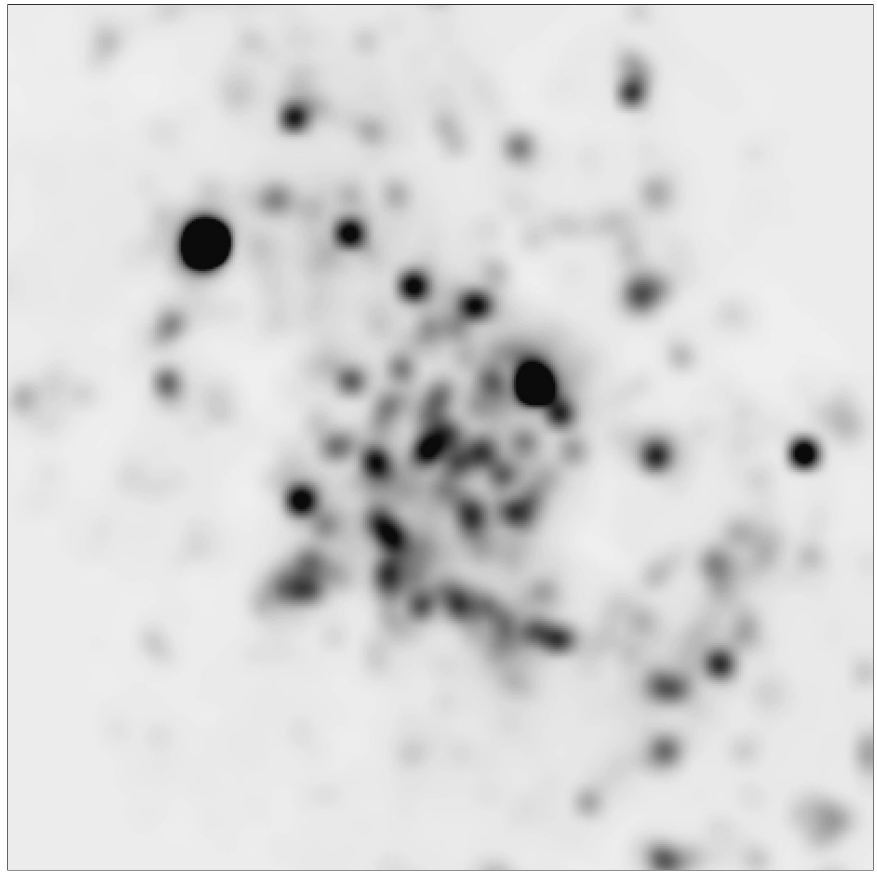

Fig. 6. $\mathrm{H} \alpha$ map of M 33 convolved to a resolution of $60^{\prime \prime}$.

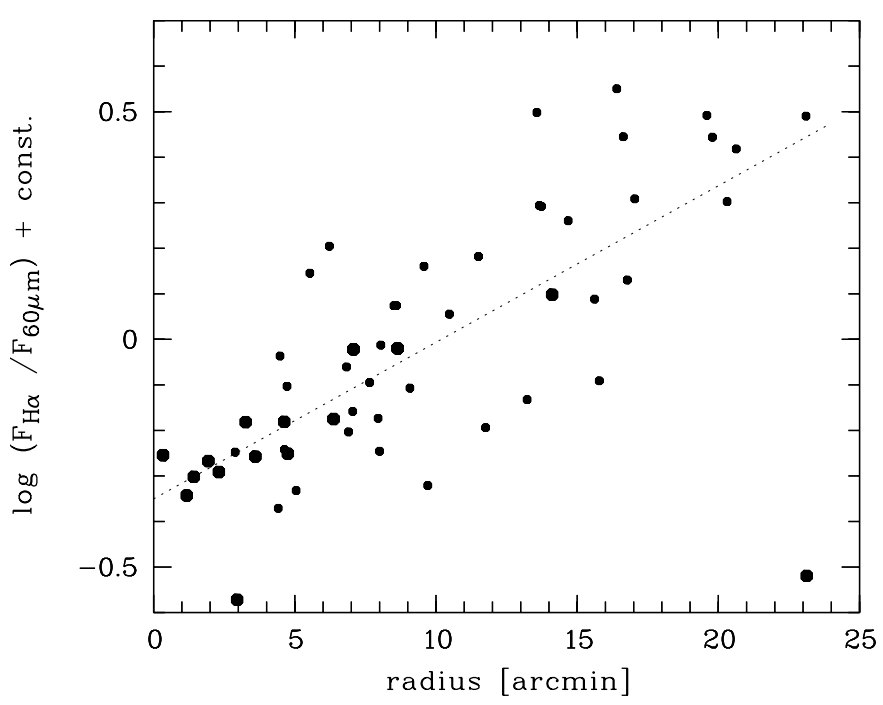

Fig. 7. $F_{\mathrm{H} \alpha} / F_{60}^{1}$ ratio for star-forming regions versus distance from the galaxy center radius. Symbol sizes indicate the brightness of the sources. (The ordinate is scaled in such a way that the mean ratio is 1.)

and $6.2 \mathrm{~cm}$ extinction values of up to $2 \mathrm{mag}$. They also found a clear decrease of the extinction for the H II regions of M 33 with increasing distance from the galaxy center of the form of $A_{V}=2.3-0.07 r$ mag (with $r$ in arcmin).

From a pixelwise comparison of the $\mathrm{H} \alpha$ emission with a $6 \mathrm{~cm}$ radio map Devereux et al. (1997) found no such trend of the internal extinction with radius but a scatter around a typical value of the order of $A_{V}=1$. They therefore assigned the increase of the $F_{\mathrm{H} \alpha} / F_{\mathrm{FIR}}$ ratio to a metallicity gradient in the sense, that hotter and more massive stars occur preferentially in the outer, metal-poorer regions of the galaxy. It seems that the radius dependence holds for the $\mathrm{H}$ II regions, but not for the diffuse emission, since, by means of multiwavelength observations of Balmer and Paschen emission lines in several H II regions, Petersen \& Gammelgaard (1997) again derived extinction values decreasing with distance from the galaxy center in a similar way as found by Israel \& Kennicutt (1980) and by Berkhuijsen (1983).

When correcting the $\mathrm{H} \alpha$ fluxes according to the above $A_{V}-r$ relation, the radial increase in $F_{\mathrm{H} \alpha} / F_{60}^{\mathrm{l}}$ mostly disappears, suggesting that this increase is mainly due to varying extinction. This suggestion is supported by the relation between extinction and cold dust radiation discussed in the next section.

There might be other processes contributing to the trend shown in Fig. 7, such as a dust depletion at locations of low gas density, or/and effects due to metallicity gradients (Devereux et al. 1997).

\subsection{The cold dust}

Analogous to the isolation of the localized emission component at 60 micron (Sect. 4.1), we now construct a map of the diffuse cold dust component by suppressing from the $170 \mu \mathrm{m}$ map the contribution from the star-forming complexes:

$$
F_{170}^{\mathrm{d}}=\frac{F_{170}-F_{60} / f^{1}}{1-f^{\mathrm{d}} / f^{1}} .
$$

The scaled difference map is shown in Fig. 8.

Since the $F_{60} / F_{170}$ ratio is higher than average in the bright knots (see Fig. 4), the difference map is overcorrected at these locations, thus yielding holes. Apart from these defects one can see that the spiral structure has almost disappeared. Instead, a disk like feature with an E-W extension of $\sim 25^{\prime}$ becomes apparent, revealing the distribution of the diffuse cold dust emission. The $170 \mu \mathrm{m}$-bright spiral arm segment in the South coincides spatially with a region of very strong $\mathrm{CO}$ emission (Lequeux, priv. comm.), probably a region of forthcoming violent star formation.

The original $170 \mu \mathrm{m}$ map shows that large amounts of dust are located in or around the star-forming regions. This cold dust associated with the massive molecular cloud complexes is expected to weaken the optical line emission of the embedded H II regions. A comparison between the $F_{\mathrm{H} \alpha} / F_{60}^{1}$ and the 170 $\mu \mathrm{m}$ flux density shows (with the exception of NGC 604) that indeed the brighter $170 \mu \mathrm{m}$ sources exhibit lower $F_{\mathrm{H} \alpha} / F_{60}$ ratios. Between the center of M 33 and a deprojected radial distance of $15^{\prime}$ the $F_{\mathrm{H} \alpha} / F_{60}^{1}$ ratio increases by a factor of about 3.3, while the surface brightness $S_{170}$ (averaged over annuli) decreases by about $120 \mathrm{MJy} \mathrm{sr}^{-1}$ over the same distance. Assuming half of the cold dust to be located in front of the H II regions, and taking this dust to be responsible for the reddening and the variation of $F_{\mathrm{H} \alpha} / F_{60}^{1}$, this gradient would imply

$$
E_{B-V} \sim 0.01 \times S_{170} \quad\left[\mathrm{MJy} \mathrm{sr}^{-1}\right] .
$$

Schlegel et al. (1998) derived from galactic cirrus extinction a similar correlation between $E(B-V)$ and the cold dust emission, $E_{B-V}=0.018 \times D T$, where $D T$ represents the IRAS $100 \mu \mathrm{m}$ flux density corrected to a reference temperature of 18.2 K using the DIRBE/IRAS maps. 


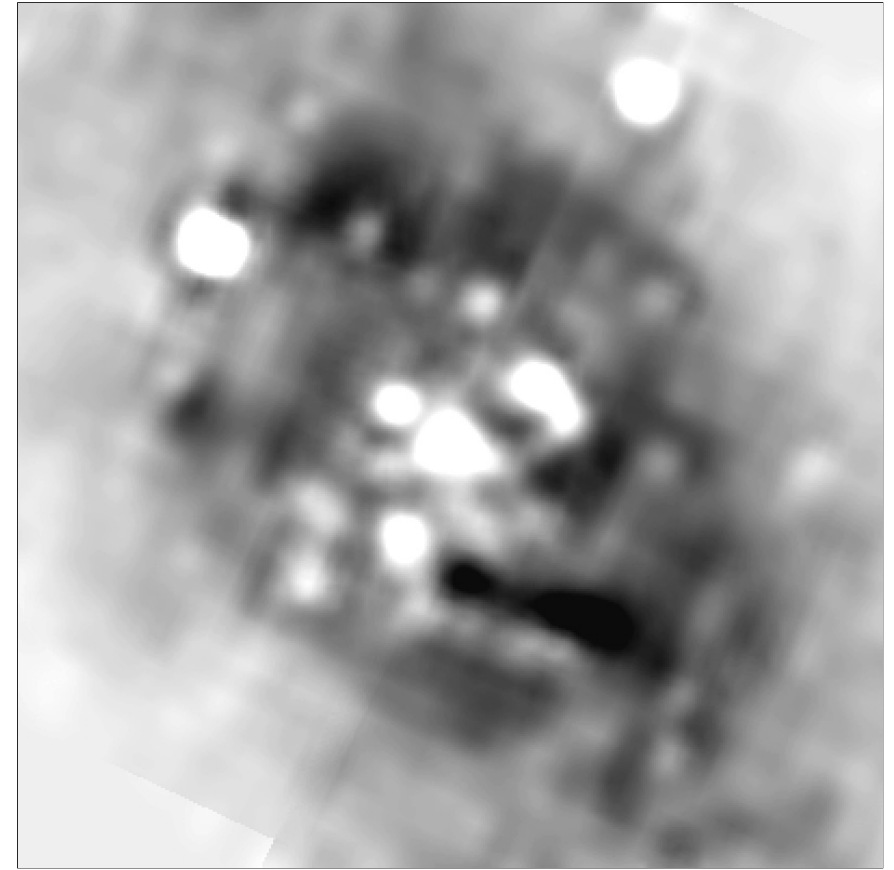

Fig. 8. Distribution of the diffuse cold dust component at $170 \mu \mathrm{m}, F_{170}^{\mathrm{d}}$, given by the scaled difference map $2\left(F_{170}-F_{60} / 0.32\right)$.

The supposition that the intrinsic extinction is traced by the cold dust would give a straightforward explanation for the apparent contradiction between Devereux et al. (1997) who find no gradient in their pixel-wise comparison of $F_{\mathrm{H} \alpha}$ and $F_{6 \mathrm{~cm}}$ (their Fig. 7) and the gradient for the $\mathrm{H}$ II regions presented by Israel \& Kennicutt (1980), since the cold dust is concentrated towards the star-forming regions as well.

The cold dust constitutes a large amount of mass in galaxies (e.g. Klaas et al. 2001; Popescu et al. 2002). Assuming an average grain size of $0.1 \mu \mathrm{m}$ and a density of $3 \mathrm{~g} \mathrm{~cm}^{-3}$, the cold dust emission in M 33 is equivalent to a mass of $6 \times 10^{6} M_{\odot}$. This is about 20\% of what is found in M 31 (Haas et al. 1998), which is expected considering the higher mass of the latter.

\subsection{Star formation}

In the following paragraphs we will discuss how far the FIR and optical star formation calibrators lead to consistent results for the whole galaxy as well as for the individual star-forming regions.

An integration of the two blackbody fit for M 33 (see Fig. 3) over the wavelength range 40 to $250 \mu \mathrm{m}$, thus including the strong diffuse cold dust component, leads to $7.6 \times$ $10^{-11} \mathrm{~W} \mathrm{~m}^{-2}=1.5 \times 10^{9} L_{\odot}$ for M 33. This is almost two times the value that one would derive from our 60 and $100 \mu \mathrm{m}$ points using Helou et al.'s (1988) relation $F_{40-120}=1.26\left(2.58 F_{60}+\right.$ $\left.F_{100}\right) 10^{-14}$ [W m ${ }^{-2}$ ], with $F_{60}$ and $F_{100}$ in Jy, which do not include the cold dust component.

Kennicutt (1998) derived from the star-burst synthesis models of Leitherer \& Heckman (1995), for solar abundances, Salpeter IMF, and assuming that the dust re-radiates all the bolometric luminosity, a relation between star-forming rate and $L_{\mathrm{FIR}}$ of $1.7 \times 10^{-10} L_{\mathrm{FIR}} M_{\odot} \mathrm{yr}^{-1} L_{\odot}^{-1}$. Since the percentage of re-radiated light for normal galaxies is only around $30 \%$ (Popescu \& Tuffs 2002b), the calibration factor used for starburst galaxies will probably underestimate the SFR in M 33. Hughes et al. (1998) derived for high redshifted HDF galaxies a calibration factor of $2.3 \times 10^{-10} L_{\mathrm{FIR}} M_{\odot} \mathrm{yr}^{-1} L_{\odot}^{-1}$, resulting in a star-forming rate of $0.35 M_{\odot} \mathrm{yr}^{-1}$ for $\mathrm{M} 33$, while the calibration of Rowan-Robinson et al. (1997) of $4.5 \times 10^{-10} L_{\mathrm{FIR}} M_{\odot} \mathrm{yr}^{-1} L_{\odot}^{-1}$ yields a value of $0.69 M_{\odot} \mathrm{yr}^{-1}$, and a recent empirical relation between $L_{\mathrm{FIR}}$ and star-forming rate (Bell 2003) gives $0.33 M_{\odot} \mathrm{yr}^{-1}$.

Applying the $F_{\mathrm{H} \alpha}-S F R$ calibration given by Kennicutt (1998) to the total $\mathrm{H} \alpha$ line luminosity of M 33, and correcting it for a mean $A_{V}=1.2$, (Israel \& Kennicutt 1980; Devereux et al. 1997) and for a mean [N II] contribution (averaged over the galaxy) of 20\% (McCall et al. 1985; Vilchez et al. 1988), the star-forming rate results to $S F R(\mathrm{H} \alpha)=0.47 M_{\odot} \mathrm{yr}^{-1}$, which is in satisfactory agreement with the FIR values.

When we, however, pick a substructure of the galaxy - for instance NGC 604, the brightest H II region in M 33 - no consistency can be achieved between the SFRs derived from the optical and from the FIR. For this example, the $\mathrm{H} \alpha$ luminosity corrected for an intrinsic extinction of $A_{V}=0.8$ (the mean of Israel \& Kennicutt 1980, and of Peterson \& Gammelgaard 1997), and for $17 \%$ [N II] contribution for NGC 604 (Vilchez et al. 1988), yields a star-forming rate of $0.046 M_{\odot} \mathrm{yr}^{-1}$. By comparison, if all the UV light were locally absorbed, its observed FIR luminosity of $5.8 \times 10^{7} L_{\odot}$ would yield SFR values of only between 0.010 (Kennicutt 1998) and $0.026 M_{\odot} \mathrm{yr}^{-1}$ (Rowan-Robinson et al. 1997), just $40 \%$ of the value derived from the optical. Thus, in this case, some $60 \%$ of the stellar UV light escapes the dust sphere around the HII region and contributes to the heating of the diffuse dust in the galaxy disk!

\subsection{A local radio-FIR correlation}

One of the tightest correlations in extragalactic astronomy is that between the FIR luminosity, as calculated from the IRAS flux densities at 60 and $100 \mu \mathrm{m}$ (for example combined in the above-mentioned relation $F_{40-120}$ by Helou et al. 1988), and the differential radio continuum luminosity in the $\mathrm{GHz}$ range for late-type galaxies. It was originally found by de Jong et al. (1985), Helou et al. (1985) and Wunderlich et al. (1987), and holds for the spatially integrated emissions of objects whose luminosity is dominated by star formation and not by an active nucleus.

This radio-FIR correlation can be theoretically explained, since the Interstellar Medium in such galaxies acts as a global calorimeter for the relativistic electrons and, in large part, for the UV photons, both of which are generated by the stars (Völk 1989). Usually the correlation is found to be somewhat steeper than unity, the radio luminosity increasing faster than linearly with the FIR luminosity. Restricting the FIR to the IRAS $60 \mu \mathrm{m}$ luminosity, Yun et al. (2001) have recently obtained a nearly linear correlation, based on a very large sample of 1809 galaxies from the local Universe, which Bell (2003) considers as a matter of conspiracy of different conflicting effects.

Our present work allows us to investigate whether such a correlation also exists for the typically sub-kpc size galactic 


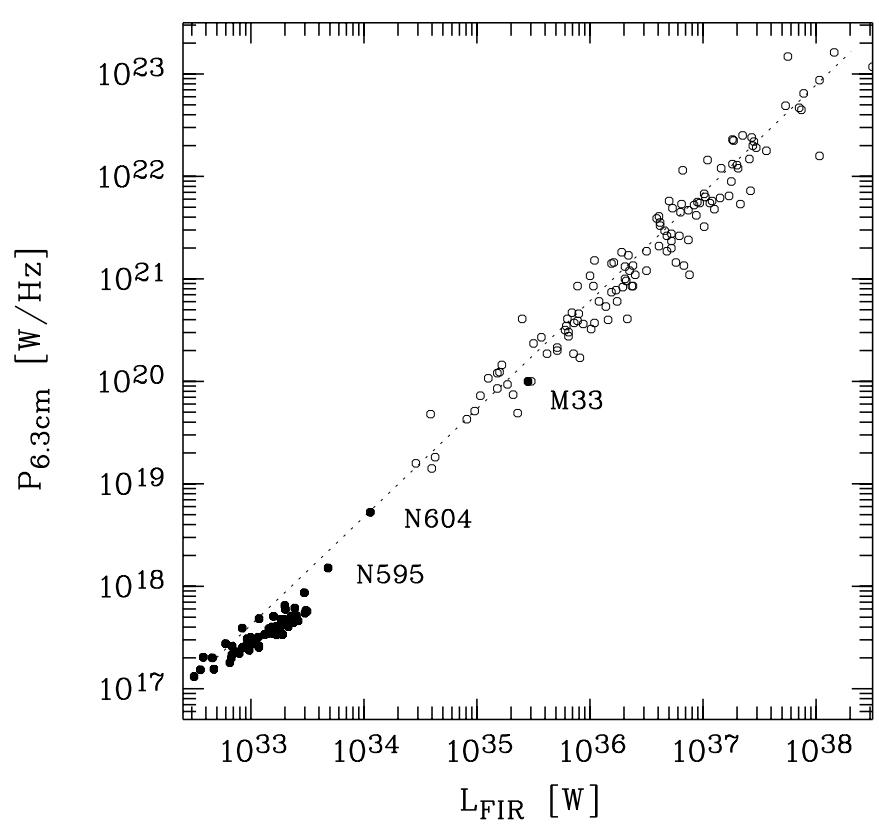

Fig. 9. Plot of the monochromatic radio luminosity versus the FIR luminosities for M 33 and its star-forming regions (filled circles) together with the data for the Effelsberg 100-m galaxy sample (Wunderlich et al. 1987, open circles). The dotted line has a slope of 1.10 (Wunderlich \& Klein 1988).

substructures such as the star-forming regions in M 33. For this purpose we made use of the $6.3 \mathrm{~cm}$ radio map of Buczilowski $\&$ Beck (1987), which has a spatial resolution of $2.4^{\prime}$. Since this resolution is considerably lower than the resolution of our 60 and $100 \mu \mathrm{m}$ maps we smoothed the FIR maps to a comparable spatial resolution, before rederiving the photometry for the knots in M 33. For the estimate of the FIR fluxes we used the relation given by Helou et al. (1988) to allow a comparison with IRAS data. Figure 9 shows the radio flux plotted versus the FIR flux. There appears to be indeed a correlation. Even taking into account the difficulties inherent in the data reduction, the slope of this correlation is smaller than unity, of the order of 0.9. We can ask ourselves why we should expect such a "local" correlation.

A priori this is not obvious. The absorption of the nonionizing stellar UV photons depends strongly on the dust column density through the star-forming regions. And even for NGC 604 about 60 percent of the UV light escapes the dust sphere of the region, as mentioned above. For smaller regions this fraction is expected to be higher. The rest goes into the diffuse dust in the galactic disk. This is the dominant FIR part in the standard global radio-FIR correlation, but it can be neglected in the "local" correlation, unless the surface filling factor of the ensemble of regions becomes a significant fraction, say more than 10 percent of the disk area. Nevertheless, the fact that we did not include the $170 \mu \mathrm{m}$ in the FIR luminosity of Fig. 9 emphasizes the shorter wavelengths, and we noted earlier that a large fraction (about $80 \%$ ) of the emission at $60 \mu \mathrm{m}$ comes from the localized component. Thus, some fraction, say $30 \%$, of the spatially integrated IRAS FIR luminosity may come from individual star-forming regions and will be proportional to their respective star formation rates. In fact, a higher star formation rate will probably imply a higher dust column density and thus a stronger than linear dependence of the FIR luminosity on the local star formation rate.

Let us next consider the radio emission from individual regions. If we assume that the sources of the radio emitting relativistic electrons are mainly the remnants of core collapse supernovae, then their progenitor stars will have remained in the star-forming region during their evolution. In addition to this non-thermal radiation, the progenitor stars will give rise to local thermal (free-free) radio emission. Therefore the necessary condition for a "local" correlation is satisfied, because the sources for both emissions are localized in the star-forming region.

Also, for a face-on galaxy, the star-forming regions are detected along the direction perpendicular to the disk. Along that direction we see the integrated emission, and the electrons confined to this column will have lost their energy by Inverse Compton and synchrotron emission, and thus their luminosity can be proportional to the local star formation rate. Much more important is the following effect: the supernova remnants which accelerate the electrons, confine them during the active remnant life time of $\sim 10^{5} \mathrm{yr}$, during which time they radiate with a very hard energy spectrum. Acceleration automatically implies confinement, because in the shock frame the particles are convected in the downstream direction. During their confinement the electrons radiate in the interior effective magnetic field which can be expected to be several times, say 5 times higher than in the average interstellar medium (Bell \& Lucek 2001; Berezhko et al. 2002). In agreement with observations (Biermann 1976; Ulvestad 1982) this implies a ratio of $\sim\left(25 \times 10^{5} \mathrm{yr}\right) /\left(3 \times 10^{7} \mathrm{yr}\right) \sim 10^{-1}$ between the synchrotron luminosity from the ensemble of star-forming regions to the diffuse luminosity from the overall galaxy where the particles have a life time of $\sim 3 \times 10^{7}$ yrs. Nevertheless, most of the relativistic electrons will leave the region after their release from the sources before having lost their energy radiatively. This is easily seen, since their radiative loss length $\left(\kappa(E) \times \tau_{\text {loss }}(E)\right)^{1 / 2} \geq$ few kpc ${ }^{1}$ exceeds the typical sizes of starforming regions, and this is even a lower limit (Ptuskin et al. 1997). Therefore, like the cold dust emission from Fig. 8, the diffuse synchrotron emission will constitute a featureless disklike structure and can be neglected in the local correlation. In fact, the escape from a star-forming region will be the more effective the higher the cold gas mass is there, and should therefore decrease with increasing FIR luminosity, if anything.

Therefore, we must expect some local radio-FIR correlation in star-forming regions, and even one which has a slope $<1$. This explains the observational results shown in Fig. 9. However, this correlation is primarily due to the confinement of synchrotron electrons in their sources (supplemented by local free-free emission), and due to local dust absorption. As such it will primarily be seen through the warm dust emission observed by IRAS. For the total FIR as measured by ISOPHOT we expect a much weaker correlation, if at all. Quantitatively,

\footnotetext{
1 Here $\kappa(E)$ is the spatial diffusion coefficient of electrons of kinetic energy $E$, and $\tau_{\text {loss }}(E)$ is the electron energy loss time. The product $\kappa(E) \times \tau_{\text {loss }}(E) \propto E^{0.1}$ is almost energy-independent.
} 
the correlation depends very much on the local transport conditions for photons and relativistic electrons and has little connection with the global radio-FIR correlation because the ingredients of the latter correlation can be neglected in the local correlation. In this picture one would predict that the radio emission responsible for the local correlation will have a hard spectrum with a spectral index flatter than about 0.5 .

\section{Conclusions}

The FIR radiation from M 33 is composed of a warm $(\sim 45 \mathrm{~K})$ and a cold $(\sim 17 \mathrm{~K})$ dust emission component, which were separated by fitting the SEDs of the entire galaxy and of individual regions with two modified Planck functions of emissivity $\beta \propto \lambda^{-2}$. We spatially isolated the two dust emission components and proved that they can be identified with separate morphological components. The warm dust is preferentially associated with the star-forming regions. The cold dust arrises in part from a diffuse disk of about $25^{\prime}$ diameter, but also has a localized component associated with the molecular clouds in star-forming regions.

Estimates of the star formation rate derived from the FIR for the whole galaxy are broadly consistent with that derived from the $\mathrm{H} \alpha$ emission. In the case of individual star-forming regions, a consistency between rates derived from the optical and from the FIR allows only a fraction of the UV radiation to be absorbed locally.

The correlation between the $F_{\mathrm{H} \alpha} / F_{60}$ ratio and the $170 \mu \mathrm{m}$ surface brightness is in agreement with the extinction relation established by IRAS/DIRBE observations for the cirrus in the Milky Way.

The ISOPHOT maps were also used to derive a local radioFIR correlation for star-forming regions in M 33. It is argued that this local correlation is due to quite different emission components than to those that lead to the well-known global radioFIR correlation.

Acknowledgements. It is a pleasure to thank Dr. Hermann-Josef Röser for carrying out the optical observations, and Drs. Rainer Beck, Eric Bell and Cristina Popescu for stimulating discussions. We also thank the referee, Dr. D. Dale, for his constructive comments which improved this paper. The ISOPHOT Data Center at MPIA are supported by Deutsches Zentrum für Luft- und Raumfahrt with funds of Bundesministerium für Bildung und Forschung, grant No. 50 QI 0201.

\section{References}

Acosta-Pulido, J. A., Gabriel, C., \& Casteneda, H. O. 2000, Experimental Astronomy, 10 (Kluwer Academic Publishers), 333

Bell, E. 2003, ApJ, 586, 794

Bell, A. R., \& Lucek, S. G. 2001, MNRAS, 321, 433

Berezhko, E. G., Ksenofontov, L. T., \& Völk, H. J. 2002, A\&A, 395, 943

Berkhuijsen, E. M. 1983, A\&A, 127, 395

Biermann, P. 1976, A\&A, 53, 295
Buczilowski, U. R., \& Beck, R. 1987, A\&ASS, 68, 171

Burstein, D., \& Heiles, C. 1982, AJ, 87, 1165

Chini, R., Kreysa, E., Krügel, E., \& Mezger, G. 1986, A\&A, 166, L8

de Jong, T., Klein., U., Wielebinski, R., \& Wunderlich, E. 1985, A\&A, 147, L6

de Vaucouleurs, G., de Vaucouleurs, A., Corwin, H. G. Jr., et al. 1991, Third Reference Catalogue of Bright Galaxies (Springer).

Devereux, N. A., Duric, N., \& Scowen, P. A. 1997, AJ, 113, 236

Gabriel, C., Acosta-Pulido, J., \& Heinrichsen, I. 1998, Astronomical Data Analysis Software and Systems VII, ASP Conf. Ser., 145, 165

Haas, M., Lemke, D., Stickel, M., et al. 1998, A\&A, 338, L33

Helou, G., Soifer, B. T., \& Rowan-Robinson, M. 1985, ApJ, 298, L7

Helou, G., Khan, I. R., Mallek, L., \& Boehmer, L. 1988, ApJS, 68, 151

Hippelein, H. H. 1986, A\&A, 160, 374

Hughes, D. H., Serjeant, S., Dunlop, J., et al. 1998, Nature, 394, 241

Israel, F. P., \& Kennicutt, R. C. 1980, ApL, 21, 1

Kennicutt, R. C. 1998, ApJ, 498, 541

Kessler, M. F., Steinz, J. A., Anderegg, M. E., et al. 1996, A\&A, 315, L27

Klaas, U., Haas, M., Müller, S. A. H., et al. 2001, A\&A, 379, 823

Laureijs, R. J., Klaas, U., Richards, P. J., et al. 2000, The ISO Handbook, Vol. V

Leitherer, C., \& Heckman, T. M. 1995, ApJS, 96, 9

Lemke, D., Klaas, U., Abolins, J., et al. 1996, A\&A, 315, L64

McCall, M. L., Rybski, P. M., \& Shields, G. A. 1985, ApJS, 57, 1

Odenwald, S., Newmark, J., \& Smoot, G. 1998, ApJ, 500, 554

Petersen, L., \& Gammelgaard, P. 1997, A\&A, 323, 697

Popescu, C. C., Misiriotis, A., Kylafis, N. D., et al. 2000, A\&A, 362, 138

Popescu, C. C., Tuffs, R. J., Völk, H. J., et al. 2002, ApJ, 567, 221

Popescu, C. C., \& Tuffs, R. J. 2002a, Rev. Mod. Astron. 15, Astronomy with Large Telescopes from Ground and Space, ed. R. E. Schielicke, Jena, Astronomische Gesellschaft, 239

Popescu, C. C., \& Tuffs, R. J. 2002b, MNRAS, 335, L41

Ptuskin, V. S., Völk, H. J., Zirakashvili, V. N., \& Breitschwerdt, D. 1997, A\&A, 321, 434

Rice, W., Boulanger, F., Viallefond, F., Soifer, B. T., \& Freedman, W. L. 1990, ApJ, 358, 418

Rowan-Robinson, M., Mann, R. G., Oliver, S. J., et al. 1997, MNRAS, 289,490

Schlegel, D. J., Finkbeiner, D. P., \& Davie, M. 1998, ApJ, 500, 525

Schmidtobreick, L., Haas, M., \& Lemke, D. 2000, A\&A, 363, 917

Schulman, E., \& Bregman, J. N. 1995, ApJ, 441, 568

Stickel, M., Lemke, D., Klaas, U., et al. 2000, A\&A, 359, 865

Tuffs, R. J., \& Popescu, C. C. 2003, in Exploiting the ISO Data Archive. Infrared Astronomy in the Internet Age, ed. C. Gry, Peschke, S., Matagne, J., et al., ESA Publications Series, ESA SP-511, 239

Tuffs, R. J., Popescu, C. C., Pierini, D., et al. 2002, ApJS, 139, 37

Tully, R. B. 1988, Nearby Galaxies Catalog (Cambridge Univ. Press)

Ulvestad, J. S. 1982, ApJ, 259, 96

Vilchez, J. M., Pagel, B. E. J., Diaz, A. I., et al. 1988, MNRAS, 235, 633

Völk, H. J. 1989, A\&A, 218, 67

Wunderlich, E., Klein, U., \& Wielebinski, R. 1987, A\&AS, 69, 487

Wunderlich, E., \& Klein, U. 1988, A\&A, 206, 47

Yun, M. G., Reddy, N. A., \& Condon, J. J. 2001, ApJ, 554, 803 